\title{
Avaliação de Mapas Conceituais e Mentais em Anatomia Humana na Medicina
}

\author{
Vera Cristina Brandão Diniz de Oliveira, Henrique Zaquia Leão, Paulo Tadeu \\ Campos Lopes
}

\author{
Programa de Pós-Graduação em Ensino de Ciências e Matemática - Universidade \\ Luterana do Brasil - Canoas-RS - Brasil \\ vcristinadiniz@gmail.com, anato.leao@gmail.com, pclopes@ulbra.br
}

\begin{abstract}
The present work aims to evaluate the use of digital technologies in the learning process by the elaboration of concept and mental maps. For that, an exploratory research of quantitative nature has been made, applying the WebQuest (WQ) as a facilitating educational strategy in the construction of student knowing with students of Applied Medical Morphology I (Human Anatomy). The results suggest that the $W Q$ was a facilitating tool in this process, reinforcing the need for improvement to its insertion in the Higher Education context.
\end{abstract}

Resumo. O presente trabalho objetiva avaliar o uso de tecnologias digitais no processo de aprendizagem por meio da elaboração de mapas conceituais e mentais. Para tanto, fez-se uma pesquisa exploratória de cunho quantitativo, aplicando a WebQuest (WQ) como estratégia educativa facilitadora na construção do saber discente com alunos da disciplina de Morfologia Médica Aplicada I (Anatomia Humana). Os resultados sugerem que a WQ foi uma ferramenta facilitadora nesse processo, reforçando a necessidade de aprimoramento para sua inserção no contexto do Ensino Superior.

\section{Introdução}

A inclusão digital deve ser trabalhada de forma coerente, sequencial e ordenada, com vistas a promover a aprendizagem de forma contextualizada. Esta precisa ser fundamentada em sequências didáticas que promovam competências e habilidades que priorizem o diálogo e o comprometimento com a disciplina proposta pelo docente [Dantas \& Oliveira 2020].

A educação problematizadora, dotada de estratégias e roteiros didáticos, é uma alternativa que busca promover aulas mais dinâmicas, exigindo uma participação mais ativa dos docentes - como educadores desta sociedade atual tecnológica - e dos educandos - como agentes da informação na construção do saber. Essa educação deve estimular criatividade, integração, crítica e autonomia. Quanto à aprendizagem de anatomia humana, foco desta pesquisa, um bom método de aprendizado e estímulo é importante para que os alunos obtenham mais conhecimento, tendo em vista que manequins, cadáveres e o uso da tecnologia são ferramentas de trabalho nessa área [Santos, Schemiguel \& Justus 2019; Albuquerque filho et al. 2020; Strini, Strini \& Bernardino Júnior 2020].

Lopes, Pereira e Silva (2013), assim como Arruda e Sousa (2014), salientam a importância de pesquisas sobre tecnologias digitais inseridas como instrumento de ensino e aprendizagem na disciplina de Anatomia Humana em cursos superiores e defendem a 
importância de problematizar a utilização das tecnologias digitais nessa disciplina. Leal (2019) reforça sobre a importância da concepção tecnológica como ferramenta inovadora imprescindível e facilitadora no que diz respeito ao acesso rápido à informação, o que contribui para os estudos práticos do corpo humano.

A anatomia é uma ciência essencialmente visual e, durante séculos, tem sido transmitida por meio de desenhos apresentados aos alunos de diversas formas. A projeção de imagens a partir de uma tecnologia como um aplicativo em mobile learning é um meio que oferece uma grande promessa para efetuar uma melhor comunicação de informações anatômicas [Trotta \& Spinillo, 2014].

Os mapas conceituais são instrumentos que desafiam os estudantes à transcrição, síntese e organização do pensamento, uma vez que representam conceitos trabalhados e exploram o potencial informativo por meio das imagens, estabelecendo importantes relações entre teoria e prática. A elaboração desses mapas favorece o desenvolvimento de múltiplas competências, tornando a ação pedagógica contextualizada e significativa [Dantas \& Oliveira 2020].

Machado e Carvalho (2019), baseados na epistemologia construtivista e na teoria de aprendizagem significativa de Ausubel (2003), descrevem que os mapas conceituais foram desenvolvidos por Joseph Donald Novak como uma ferramenta para representar e organizar o pensamento. A experiência pessoal como psicólogo e professor permitiu que Ausubel refletisse sobre caminhos a serem oferecidos para que a percepção, compreensão e elaboração fossem facilitadas no processo de ensino. Em razão disso, o pesquisador defende que o estudante consegue desenvolver um papel de importância fundamental na designação de significados, agregando com aptidão os conteúdos da sala de aula aos seus conhecimentos prévios, aprendendo de modo correto e necessário [Masini \& Moreira 2017; Fonseca \& Salvi 2019).

A visão cognitivista, na formação médica, com uma estruturação educativa bem elaborada, é um dos requisitos fundamentais para que um indivíduo consiga desenvolver uma prática reflexiva e aplicar salutarmente o que foi aprendido. Com base nisso, acredita-se que os mapas conceituais são ferramentas importantes para a avaliação da estruturação de conhecimento dos alunos da graduação de Medicina, uma vez que objetiva a implementação do saber [Utagawa, Ribeiro \& Bianconi 2013].

Os mapas conceituais podem beneficiar os estudos do Ensino Superior de diferentes maneiras, tais como: promover a aprendizagem dos conteúdos, desenvolver o pensamento crítico, relacionar a teoria e a prática e ajudar a resolver problemas. Essa técnica é utilizada para estabelecer relações entre conceitos e sistematizar o conhecimento significativo [Okada 2008]. É considerada uma importante ferramenta educacional, visto que promove o desenvolvimento cognitivo no Ensino Superior e aprimora a formação acadêmica, que demanda pensamento crítico, capacidade de resolução de problemas, compreensão de conceitos e apreensão do conhecimento [Gomes et al. 2011; Moreira 2013; Machado \& Carvalho 2019].

O uso de mapas conceituais é salutar ferramenta na relação entre aulas teóricas e práticas, sendo um instrumento estratégico para o ensino da Medicina. Eles potencializam a construção do conhecimento, enfatizando particularmente a educação e formação médicas, que demandam técnicas, métodos e instrumentos que promovam a aprendizagem [Gomes et al. 2011; Moreira 2013; Machado \& Carvalho 2019]. Os mapas mentais permitem registrar o pensamento de maneira criativa e flexível, permitindo 
trabalhar a essência de um conceito, que com o auxílio de imagens e ideias relacionadas favorecem o processo criativo e não linear de organização da informação proposta (Lima \& Manini 2016; Ribeiro, Linhares \& Chagas, 2017).

Com base no contexto apresentado, a pergunta que norteou este trabalho foi: as WebQuests ( $W Q$ ) introduzidas na disciplina de Anatomia Humana propiciarão rendimento cognitivo e aprendizagem significativa? Nessa perspectiva, o trabalho teve por objetivo avaliar o uso de tecnologias digitais no processo de ensino e aprendizagem de anatomia humana em cursos superiores da área da saúde, construindo um Ambiente Virtual de Aprendizagem (AVA) estruturado com sequências didáticas.

\section{Metodologia}

Foi realizada uma pesquisa exploratória de cunho quantitativo para avaliar o efeito do uso da ferramenta $W Q$ e a correspondente percepção de melhorias na qualidade do ensino em anatomia humana.

A ferramenta $W Q$ foi disponibilizada na plataforma Google Sites com os seguintes componentes: Introdução, Tarefa (onde houve a solicitação da construção dos mapas mentais e conceituais), Processo, Recursos e Avaliação (onde foi disponibilizada a explicação sobre a construção de mapas que deveriam abranger dois sistemas corporais) [Fraiha-Martins \& Gonçalves 2018].

A metodologia WebQuest é uma proposta de trabalho concebida e implantada por professores para ser resolvida por alunos, sendo que a informação com que estes interagem é proveniente, em parte ou na totalidade, de recursos disponíveis on-line. Essa metodologia é um incentivo para que outros professores do Ensino Superior valorizem essa estratégia como forma de proporcionar aos seus alunos formas de aprendizagem ativa, colaborativa e, principalmente, alinhadas à utilização de recursos atualizados [Barros \& Gregório 2018].

A pesquisa ocorreu durante o segundo semestre de 2018 (35 alunos) e primeiro semestre de 2019 (31 alunos) na disciplina de Morfologia Médica Aplicada I do curso de Medicina de uma universidade privada da Região Metropolitana de Porto Alegre-RS. A amostra foi selecionada por conveniência, sendo composta por acadêmicos matriculados no período citado. Como critérios de inclusão, foram definidos: acadêmicos dos períodos referidos do curso médico que estavam presentes no momento da coleta de dados e assinatura do Termo de Consentimento Livre e Esclarecido (TCLE), sendo excluídos os acadêmicos ausentes durante a coleta de dados.

Em relação à avaliação dos mapas mentais e conceituais, foram observados o progresso do aluno e sua evolução no processo de aprendizagem, considerando sua capacidade de construção, coerentemente ao conteúdo exposto.

Os mapas mentais e conceituais foram avaliados e analisados de acordo com critérios e descrições adaptadas em Silva $(2014 ; 2015)$. As análises quantitativas e os dados identificatórios foram feitos com base nas ferramentas da estatística descritiva.

\section{Resultados e discussão}

A partir da análise das Tabelas 1 e 2, a seguir, observa-se que, dentre todos os mapas analisados, $53 \%$ dos mapas confeccionados foram mapas mentais, enquanto $46,9 \%$ foram mapas conceituais. Dessa forma, mesmo contando com quantidades semelhantes de cada 
modalidade de mapa, pode-se observar que os alunos tiveram maior afinidade na elaboração dos mapas mentais. É relevante destacar que existe uma tendência na elaboração do mesmo tipo de mapa pelo mesmo aluno, sendo que apenas um dos 66 alunos analisados optou por confeccionar um mapa mental e um mapa conceitual.

Tabela 1. Índice de notas e quantidade de mapas mentais (MM) e mapas conceituais (MC) elaborados em 2018/2

\begin{tabular}{|c|c|c|c|c|c|}
\hline Notas & $\begin{array}{c}\text { Quantidade de } \\
\text { MM com notas }\end{array}$ & $\begin{array}{c}\text { Quantidade de } \\
\text { MC com notas }\end{array}$ & Mapas & $\begin{array}{c}\text { \% Nota } \\
\text { mínima }\end{array}$ & $\begin{array}{c}\text { \% Nota } \\
\text { máxima }\end{array}$ \\
\hline 1 & - & - & - & - & - \\
\hline 2 & - & - & - & - & - \\
\hline 3 & - & - & - & - & - \\
\hline 4 & - & - & - & - & - \\
\hline 5 & 4 & 6 & - & - & - \\
\hline 6 & 2 & 4 & - & - & - \\
\hline 7 & 2 & 2 & - & - & - \\
\hline 8 & 2 & 9 & - & - & - \\
\hline 9 & 3 & 2 & - & - & - \\
\hline 10 & 19 & 15 & - & - & - \\
\hline Total & $\mathbf{3 2}$ & $\mathbf{3 8}$ & $\mathbf{7 0}$ & $\mathbf{1 4 , 2}$ & $\mathbf{4 8 , 5}$ \\
\hline
\end{tabular}

Com base na Tabela 1 , conclui-se que $48,5 \%$ dos alunos obtiveram nota máxima na avaliação dos mapas, enquanto $14,2 \%$ obtiveram a nota mínima, demonstrando que a maioria desempenhou a atividade da maneira esperada, o que reflete construção dos saberes a partir de conteúdos assimilados durante a jornada acadêmica. Os indicadores podem ser utilizados a fim de tecer uma avalição sob a forma de parecer avaliativo ou numérico.

Tabela 2. Índice de notas e quantidade de mapas mentais (MM) e mapas conceituais (MC) elaborados em 2019/1

\begin{tabular}{|c|c|c|c|c|c|}
\hline Notas & $\begin{array}{c}\text { Quantidade de } \\
\text { MM com notas }\end{array}$ & $\begin{array}{c}\text { Quantidade de } \\
\text { MC com notas }\end{array}$ & Mapas & $\begin{array}{c}\text { \% Nota } \\
\text { mínima }\end{array}$ & $\begin{array}{c}\text { \% Nota } \\
\text { máxima }\end{array}$ \\
\hline 1 & - & - & - & - & - \\
\hline 2 & - & - & - & - & - \\
\hline 3 & - & - & - & - & - \\
\hline 4 & - & - & - & - & - \\
\hline 5 & 1 & 4 & - & - & - \\
\hline 6 & 4 & 2 & - & - & - \\
\hline 7 & 1 & 0 & - & - & - \\
\hline 8 & 5 & 1 & - & - & - \\
\hline 9 & 11 & 3 & - & - & - \\
\hline 10 & 16 & 14 & - & - & - \\
\hline Total & $\mathbf{3 8}$ & $\mathbf{2 4}$ & $\mathbf{6 2}$ & $\mathbf{8}$ & $\mathbf{4 8 , 3}$ \\
\hline
\end{tabular}

De acordo com a Tabela 2, 48,3\% dos alunos obtiveram nota máxima na avaliação dos mapas, enquanto $8 \%$ obtiveram a nota mínima, tendo ainda menos alunos com a nota mínima em relação à turma apresentada na Tabela 1 . Isso pode ter relação com o maior preparo atribuído aos pesquisadores após a realização da pesquisa com a primeira turma, podendo ter aprimorado sua metodologia de aplicação da atividade no decorrer do estudo, demonstrando que tanto os alunos quanto os docentes conseguem aprimorar suas práticas educacionais.

Para ambas as turmas analisadas, os mapas classificados como ideais demonstraram hierarquia entre os conteúdos propostos, eram legíveis por não apontarem 
erros ortográficos, estavam precisos na aplicação de conceitos anatômicos, completos por apresentar um número suficiente de abordagens, além de bem relacionados e sofisticados no que tange à significância das conexões e da qualidade de ilustração. Os avaliados nos mesmos parâmetros mencionados foram mensurados de forma mínima por não demonstrarem hierarquia entre os conteúdos propostos, serem pouco legíveis e apontarem erros ortográficos, além de não estarem precisos na aplicação de conceitos anatômicos, incompletos por apresentar um número insuficiente de abordagens mal relacionadas e não sofisticadas em relação à significância das conexões e qualidade de ilustração. Segundo Novak e Cañas (2010), o novo modelo de educação leva à criação de portfólios de conhecimento individual que servem como alicerce para futuras aprendizagens.

Avaliaram-se os mapas mentais e conceituais de forma mais simples, sendo mensurados de acordo com critérios e descrições adaptados dos modelos de Novak e Gowin (1984) e Mueller (2014), descritos em Silva (2015), porém de maneira a não desestimular o aluno em função de nota mínima prevista de 1,0 ponto e máxima de 5,0 pontos, respondendo aos critérios estabelecidos pelos autores (Tabela 3 ).

Apesar de existirem modelos propostos, nada impede que um profissional da área de educação elabore seu próprio constructo e/ou modelo de avaliação, que partirá das expectativas que ele tem de seus estudantes [Silva 2015]. Todos os modelos consideram alguns parâmetros em comum para avaliação, como: organização progressiva dos conceitos, rede de relação conceitual e/ou ideias construídas, palavras e/ou frases conectivas e cognitivas e aspectos visuais de comunicação por meio dessa ferramenta. Indiferentemente do modelo de avaliação que os docentes optem por aplicar, cabe dizer que eles devem ajudar os discentes a aperfeiçoarem seus mapas e, consequentemente, os seus conhecimentos referentes a determinado conteúdo [Silva 2014].

Tabela 3. Modelo de avaliação de mapas mentais e conceituais, adaptado e sugerido pelos autores

\begin{tabular}{|c|c|}
\hline Critério & Descrição \\
\hline Hierarquia & Existe hierarquia entre os conteúdos. Atribui-se $(1,0$ a 2,0$)$ pontos. \\
\hline Legível & Erros de ortografia: atribui-se 1,0 a 2,0 pontos. \\
\hline Preciso & $\begin{array}{l}\text { Conceitos utilizados com precisão: } \\
\text { - } \quad \text { pouca }-1,0 \text { ponto; } \\
\text { - } \quad \text { muita }-2,0 \text { pontos. }\end{array}$ \\
\hline Completo & $\begin{array}{l}\text { Número suficiente de conceitos, bem relacionados: } \\
\text { - } \quad \text { uso limitado }-1,0 \text { ponto; } \\
\text { - } \quad \text { suficiente }-2,0 \text { pontos. }\end{array}$ \\
\hline Sofisticado & $\begin{array}{l}\text { Conexões significativas - em se tratando de MM, a qualidade da ilustração: } \\
\text { - } \quad \text { pouca ou nenhuma }-1,0 \text { ponto; } \\
\text { - } \quad \text { significativo e original }-2,0 \text { pontos. }\end{array}$ \\
\hline Pontuação & $\begin{array}{l}\text { Pode ser mínima, de } 5,0 \text { até } 10,00 \text { pontos. } \\
\text { Obs.: a não entrega justificada e não realização denota nota } 0,0 \text { ponto. }\end{array}$ \\
\hline
\end{tabular}

Fonte: Adaptado de Silva $(2014 ; 2015)$.

Os critérios expostos na Tabela 3 contemplam avaliar mapas mentais e mapas conceituais. Parâmetros que não zeram a avaliação justificam 0,5 pontos ofertados como extra na avaliação final da disciplina. 
Silva $(2014 ; 2015)$ sugere sete autores como parâmetros de modelos avaliativos de mapas conceituais. Mc Murray (2014), baseado no modelo de Novak e Gowin (1984), considera como um dos critérios de avaliação o item "inaceitável", assim como a University of Minnesota Digital Media Center (2004), que descreve como "inadequado". O modelo do National Computation Science Education Consortium (NCSEC, 2000) utiliza a expressão "abaixo das expectativas"; e Bartels (1995) descreve que existem mapas que devem ser zerados por não se adequarem ao conteúdo. Os modelos de Mueller (2007), Cronin, Dekker e Dunn (1982) e Bartels (1995) se assemelham ao de Novak e Gowin (1984), denominado de critério de pontuação de mapas conceituais, e sugerem avaliar sem desprezar os aspectos cognitivos de construção.

É importante que se tenha o cuidado de observar a subjetividade e cognição de cada sujeito no momento da construção, oportunizando refazer e refletir sobre uma construção mais adequada, embasada na objetividade de apreensão de conhecimentos e conteúdos propostos na disciplina, portanto, evitando termos "punitivos" e "inibidores". Segundo Silva (2015), cada professor ou instituição pode adaptar ou até mesmo conceber o seu próprio modelo.

Para facilitar a compreensão da análise dos mapas mentais e conceituais, foram escolhidos cinco critérios para classificação, tendo como base os mapas produzidos pelos alunos - um exemplo de cada tipo para a explicação. Com relação ao primeiro critério, foram selecionados dois mapas produzidos pelos alunos para diferenciá-los e exemplificados modelos de mapas que contemplaram todos os critérios estabelecidos e outros que atenderam à demanda mínima, lembrando a possibilidade de retomada pelo aluno, além da aceitação de seu trabalho, respeitando sua subjetividade cognitiva. Cabe ressaltar que não houve diferenciação quanto à avaliação pelo fato de se tratar de layout manuscrito ou digital, sendo apenas avaliados de acordo com os critérios estabelecidos, conforme referido anteriormente.

Silva (2015) expõe sobre o grande desafio de avaliar, devido ao fato de serem elaborados com a base cognitiva de cada sujeito. Deve ser observado o progresso do aluno, sua evolução no processo da aprendizagem e sua capacidade de construção, coerentemente ao conteúdo exposto para evitar a mera crítica pontual e desestimuladora. Não há mapa certo ou errado, e sim categorizado, não cabendo debater ou discutir sobre o modelo ideal. Considera-se a especificidade e a subjetividade de cada curso superior que deseja implantá-lo.

Enquanto o mapa conceitual trabalha várias ideias progressivas para se chegar a um conceito, o mapa mental percorre vários caminhos direcionados por uma ideia [Silva 2015]. Em relação à temática abordada, entende-se a importância de explorar diferentes maneiras de aprimorar o processo de ensino e aprendizagem no Ensino Superior, no qual o professor mediador deve incrementar suas aulas com a utilização de meios digitais, desenvolvendo competências e habilidades científicas. É fundamental que o docente conheça e acompanhe as dificuldades dos discentes, a fim de buscar meios de amenizá-las ou superá-las.

\section{Considerações finais}

As atividades on-line podem estimular os alunos na construção do conhecimento, favorecendo a efetivação do aprendizado. Enfatiza-se a importância de explorar 
diferentes ferramentas no processo de ensino e aprendizagem no Ensino Superior, no qual o professor mediador deve incrementar suas aulas com meios digitais, desenvolvendo competências e habilidades científicas por intermédio da utilização de WebQuests na disciplina de Anatomia Humana para promoção de rendimento cognitivo e aprendizagem significativa.

A avaliação quanto ao uso de tecnologias digitais no processo de ensino e aprendizagem de anatomia humana promoveu a elaboração de mapas que indicam colaborar na construção do conhecimento e aprendizagem científica. Percebeu-se que o uso de mapas conceituais no planejamento da ementa de uma disciplina ajuda a tornar o ensino contextualizado, contribuindo para melhorar o desempenho, assim como facilita a construção do aprendizado, reforçando o Ensino Superior incrementado por meio de práticas disponibilizadas pelas mídias digitais. Mediante os resultados, afirma-se que essa metodologia de ensino poderia ser amplamente difundida no Ensino Superior, uma vez que demonstrou adesão, interesse e resultados positivos por parte dos alunos na confecção da atividade, levando em conta que $100 \%$ dos alunos realizaram a proposta.

Agradecimentos e apoios: $O$ presente trabalho foi realizado com o apoio da Coordenação de Aperfeiçoamento de Pessoal de Nível Superior - Brasil (CAPES) Código de Financiamento 001.

\section{Referências}

Albuquerque Filho, E. T. et al. (2020). Aplicação de metodologias ativas de ensino no estudo da anatomia humana frente ao modelo tradicional. Brazilian Journal of Health Review, 3(2), 1457-1464.

Arruda, R. M., \& Sousa, C. R. A. (2014). Aproveitamento teórico-prático da disciplina Anatomia Humana do curso de Fisioterapia. Revista Brasileira de Educação Médica, 38(1), 65-71.

Ausubel, D. P. (2003). Aquisição e retenção de conhecimentos: uma perspectiva cognitiva. Lisboa: Plátano.

Barros, R. R., \& Gregório, J. S. (2018). WebQuest no ensino de biologia: um estudo de caso com alunos do Ensino Médio. Ciclo Revista: Experiências em Formação no IF Goiano, 3(1).

Dantas, A. T. S., \& Oliveira, M. T. (2020). Construção e aplicação de uma sequência didática utilizando o smartphone como recurso tecnológico para o ensino de Biologia. REAMEC-Rede Amazônica de Educação em Ciências e Matemática, 8(1), 195-211.

Fonseca, R. L., \& Salvi, R. F. (2019). A aprendizagem significativa no Ensino Superior: o uso de mapas conceituais na formação do professor de geografia. Geografia Ensino \& Pesquisa, 23.

Fraiha-Martins, F. F., \& Gonçalves, T. V. O. (2018). Processos de letramento científicodigital: uso de WebQuest na formação inicial de professores. Educação \& Linguagem, 21(1), 75-93. 
Gomes, A. P. et al. (2011). O papel dos mapas conceituais na educação médica. Revista Brasileira de Educação Médica, 35(2), 275-282.

Leal, T. et al. (2019). O uso da tecnologia em prol do aprendizado em anatomia humana. Anais do $11^{\circ}$ Salão Internacional de Ensino, Pesquisa e Extensão da UNIPAMPA: Salão de Ensino. Santana do Livramento-RS/Rivera (Uruguai).

Lima, J. L. O., \& Manini, M. P. (2016). Metodologia para análise de conteúdo qualitativa integrada à técnica de mapas mentais com o uso dos softwares Nvivo e Freemind. Informação \& Informação, 21(3), 63-100.

Lima, J. M. P. (2019). A importância da sequência didática para a aprendizagem significativa da matemática. Artigos.Com, 2, e829-e829.

Lopes, R. T., Pereira, A. C., \& Silva, M. A. D. (2013). O uso das TIC no ensino da morfologia nos cursos de saúde do Rio Grande do Norte. Revista Brasileira de Educação Médica, 37(3), 359-364.

Machado, C. T., \& Carvalho, A. A. (2019). Os efeitos dos mapas conceituais na aprendizagem dos estudantes universitários. ETD-Educação Temática Digital, 21(1), 259-277.

Masini, E. F. S., \& Moreira, M. A. (2017). Aprendizagem significativa na escola. Curitiba, PR: CRV.

Moreira, M. A. (2013). Aprendizagem significativa, organizadores prévios, mapas conceituais, diagramas $V$ e unidades de ensino potencialmente significativas. Curitiba. (Material de apoio para o curso Aprendizagem Significativa no Ensino Superior: Teorias e Estratégias Facilitadoras). PUCPR, Curitiba.

Novak, J. D. \& Cañas, A. J. (2010). A teoria subjacente aos mapas conceituais e como elaborá-los e usá-los. Práxis Educativa, 5(1), 9-29.

Okada, A. (2008). O que é cartografia cognitiva e por que mapear redes de conhecimento. In A. Okada. Cartografia cognitiva: mapas do conhecimento na pesquisa, aprendizagem e formação docente (pp. 37-65). Cuiabá: Editora KCM.

Ribeiro, V. M. T. F., Linhares, R. N., \& Chagas, A. M. (2017). Mapas mentais e seu impacto na produção e raciocínio crítico-criativos na percepção/formação de alunos e professores de uma Escola Técnica em Sergipe. Anais do Simpósio Internacional de Educação e Comunicação - SIMEDUC. Aracaju, SE. Recuperado de https://eventos.set.edu.br/index.php/simeduc/article/view/8572

Santos, K. A. S. A., Schemiguel, K., \& Justus, J. F. C. (2019). Utilização de Tecnologias de Informação e Comunicação (TICs) no Ensino e Aprendizagem de Anatomia Humana. Brazilian Journal of Development, 5(11), 25172-25176.

Silva, E. C. (2015) Mapas conceituais: propostas de aprendizagem e avaliação. RAEP Administração: Ensino e Pesquisa, 16(4), 785-815.

Silva, E. C. (2014). Mapas conceituais: modelos de avaliação. Anais do $6^{\circ}$ International Conference on Concept Mapping. Santos, SP. Recuperado de https://www.researchgate.net/publication/273717843_MAPAS_CONCEITUAIS_M ODELOS_DE_AVALIACAO 
Strini, P. J. S. A., Strini, P. J. S. A., \& Bernardino Júnior, R. (2020). Metodologia ativa em aulas práticas de anatomia humana: a conjunta elaboração de roteiros. Ensino em Re-Vista, 27(2), 680-697.

Trotta, T., \& Spinillo, C. G. (2014). Tecnologias no aprendizado da Anatomia Humana: possíveis contribuições para o ensino da medicina. Revista Brasileira de Design da Informação, 11(1), 1-20.

Utagawa, C. Y., Ribeiro, R., \& Bianconi, M. L. (2013). Avaliação de mapas conceituais e estrutura de conhecimento em acadêmicos de medicina. Práxis, 5(9). 